$43-48$

\title{
Spinal fractures in recreational bobsledders: an unexpected mechanism of injury
}

Authors Erik P Severson ${ }^{1}$, D'Mitri A Sofianos ${ }^{1}$, Amy Powell ${ }^{1}$, Michael Daubs ${ }^{1}$, Rakesh Patel ${ }^{2}$, Alpesh A Patel ${ }^{3}$

Institutions ${ }^{1}$ Department of Orthopaedics, University of Utah School of Medicine, Salt Lake City, Utah, USA

${ }^{2}$ Department of Orthopaedic Surgery, University of Michigan School of Medicine, Ann Arbor, MI, USA

${ }^{3}$ Department of Orthopaedic Surgery and Rehabilitation, Loyola University Stritch School of Medicine, Salt Lake City, Utah, USA

\section{ABSTRACT}

Study design: Retrospective case series and literature review.

Objective: To report and discuss spinal fractures occurring in recreational bobsledders.

Summary of background data: Spinal fractures have been commonly described following traumatic injury during a number of recreational sports. Reports have focused on younger patients and typically involved high-impact sports or significant injuries. With an aging population and a wider array of recreational sports, spinal injuries may be seen after seemingly benign activities and without a high-impact injury.

Methods: A retrospective review of two patients and review of the literature was performed.

Results: Two patients with spinal fractures after recreational bobsledding were identified. Both patients, aged 57 and 54 years, noticed a simultaneous onset of severe back pain during a routine turn on a bobsled track. Neither was involved in a high-impact injury during the event. Both patients were treated conservatively with resolution of symptoms. An analysis of the bobsled track revealed that potential forces imparted to the rider may be greater than the yield strength of vertebral bone.

Conclusions: Older athletes may be at greater risk for spinal fracture associated with routine recreational activities. Bobsledding imparts large amounts of force during routine events and may result in spinal trauma. Older patients, notably those with osteoporosis or metabolic bone disease, should be educated about the risks associated with seemingly benign recreational sports. 


\section{INTRODUCTION}

Spine injuries related to recreational participation in athletic activities have been well described in the literature [1-4]. Common spinal injuries that occur include muscle strains, muscle spasms, disc herniations, as well as vertebral body compression and avulsion fractures [1]. Spinal injuries have been described in football, hockey, wrestling, diving, skiing, snowboarding, rugby, cheerleading, baseball, and golf $[1,4,5]$. The literature, however, is typically focused on a young patient population often with an emphasis on catastrophic injuries that have a clearly identifiable traumatic mechanism.

This article reports spinal injuries occurring in two older patients during a recreational bobsled run. In addition, the variables that contribute to the increased propensity of fractures in the aging spine are discussed. After reviewing the forces imparted on the spine and the increased chances of spine fracture in the elderly, proposed safety guidelines are recommended for recreational bobsledders. To our knowledge, this is the first discussion in the literature to date on bobsled-related fractures of the spine.

\section{CASE REPORTS}

The two cases presented involve a retired 57-year-old man (patient 1) and his 54-year-old wife (patient 2). Neither patient had any history of fractures, medical comorbidities, osteoporosis, or nicotine use. They are presented together due to the similarity between the two cases. Both patients have consented to the publication of their case histories. While on vacation, the couple elected to ride a local bobsled track previously used in international competitions. They had an experienced driver navigate the bobsled down a 4380 foot track. During the run, they each simultaneously described an immediate sharp, stabbing pain in their back while in the middle of a specific turn. There was no rollover, ejections, collisions, or any unusual occurrences during the ride. The bobsled was reportedly under control at all times. After the run had been completed, the two patients were brought to our institution for evaluation of severe back pain. Neither patient had experienced numbness, tingling or loss of bowel or bladder function. Both demonstrated strong and symmetric motor examinations with no sensory deficits, normal rectal tone, and no pathological reflexes. Patient 1 demonstrated paraspinal tenderness at the thoracolumbar junction and patient 2

Fig 1a-b Sagittal (a) and axial (b) CT images of Patient 1 demonstrate a compression fracture of T12 with anterior column.
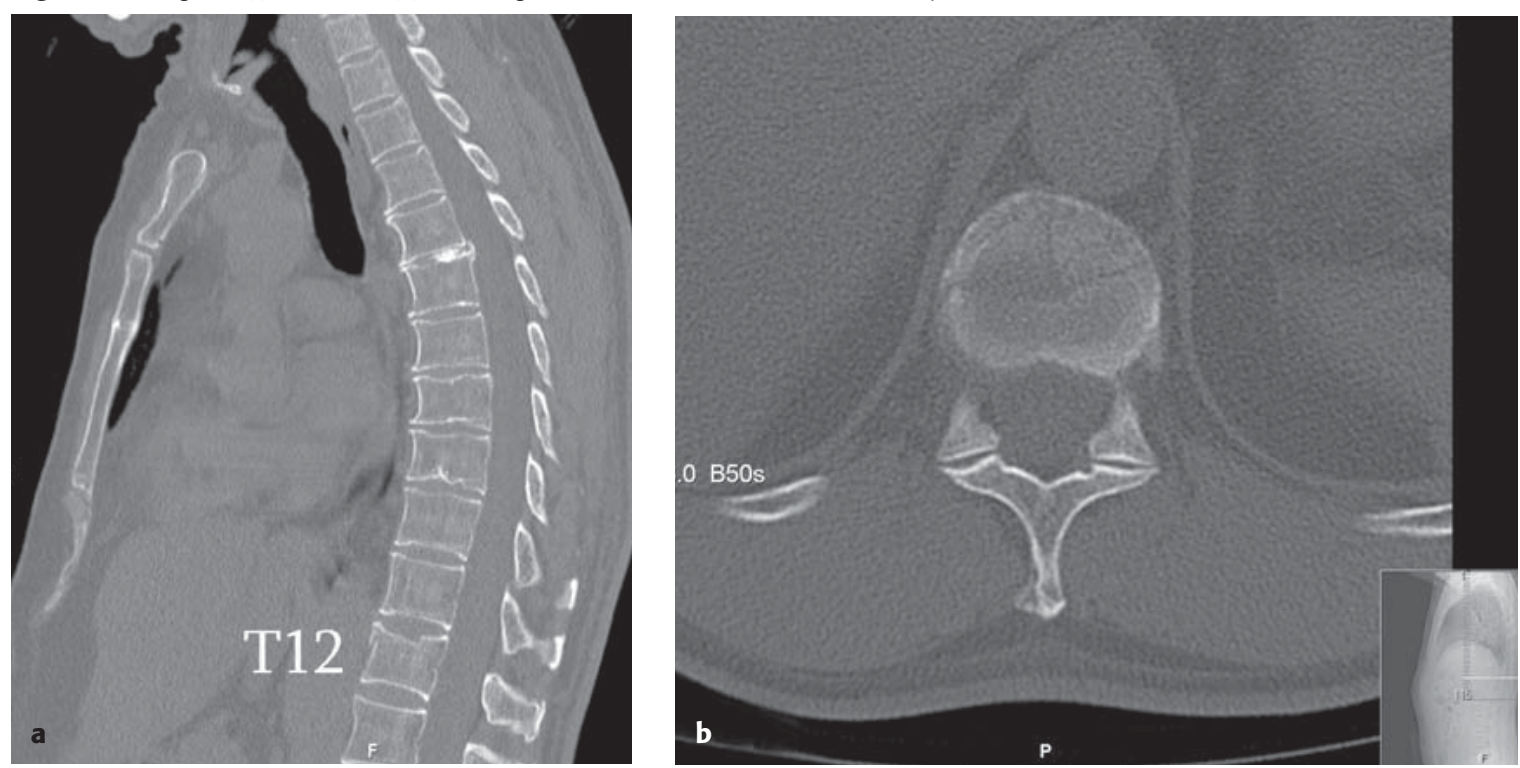
had midline tenderness but no palpable gap or step-offs at the thoracolumbar junction.

$\mathrm{X}$-rays and computed tomographic (CT) images revealed that patient 1 had an isolated T12 compression fracture

(Fig 1) and patient 2 had an isolated T12 burst fracture

(Fig 2). The burst fracture was associated with less than $10 \%$ canal compromise, no sagittal or coronal deformity, and an intact posterior ligamentous complex. Injuries were classified according to the Thoracolumbar Injury Classification and Severity Score (TLICS) [6, 7]. The TLICS scores for patient 1 and patient 2 was 1 and 2 , respectively. They were both treated nonoperatively with the use of a thoracolumbar orthosis (TLSO).

After passing a TLSO brace-trial, upright x-rays were obtained verifying stable alignment of the spinal fractures. Both patients were discharged and returned home to England within 2 weeks of their injuries. After 6 weeks of bracing, $x$-rays were obtained by their primary care provider demonstrating stable alignment. The orthoses were discontinued and a physiotherapy program was initiated. The patients had also independently sought chiropractic care after that time. Through direct communications with the patients, 24-month follow-up was obtained. Their pain scores improved steadily, with a current Visual Analog Scale (VAS) rating at $2 / 10$ for both patients. They reported no functional limitations from their injuries and a return to normal daily and recreational activities. Osteoporosis evaluation was requested but deemed unnecessary by their primary care provider due to their age and low number of risk factors.

\section{DISCUSSION}

Bobsledding began in the late 19th century in Switzerland and is now recognized as an international sport. The particular track that the two patients rode is 4380 feet in length, displays a vertical drop of 340 feet, and has a slope of $7.8 \%$. The sled itself weighs approximately $600 \mathrm{lbs}$ and will reach maximum speeds of 80 miles per hour. Injuries associated with bobsledding have not been previously described in detail.

Given the nature of bobsledding, it would be assumed that injuries occur during overturns, collisions, or ejections. In reviewing these cases, it is unique that both middle-aged individuals suffered thoracic fractures without any overt traumatic event. The determinants of fracture, therefore, include the forces imparted by the bobsled ride as well as the mechanical and biological status of the patient's bone (eg, osteopenia, osteoporosis).

\section{Vertebral fracture risk}

In a finite element model, Imai et al [8] found vertebral compression yield strength to be $2154 \mathrm{~N}( \pm 685 \mathrm{~N})$. This value is similar to values found in other biomechanical studies and can be considered the failure load in an aging, osteopenic, or osteoporotic spine [9, 10]. Silva [9] defined fracture risk based on applied load and load to failure by the equation:

Factor of Risk $(\Phi)=$ Applied Load / Fracture Load

Fig 2a-b Sagittal (a) and axial (b) CT images of Patient 2 demonstrate a burst fracture of $\mathrm{T} 12$ with $<10 \%$ canal stenosis, normal spinal alignment, and no posterior column involvement.
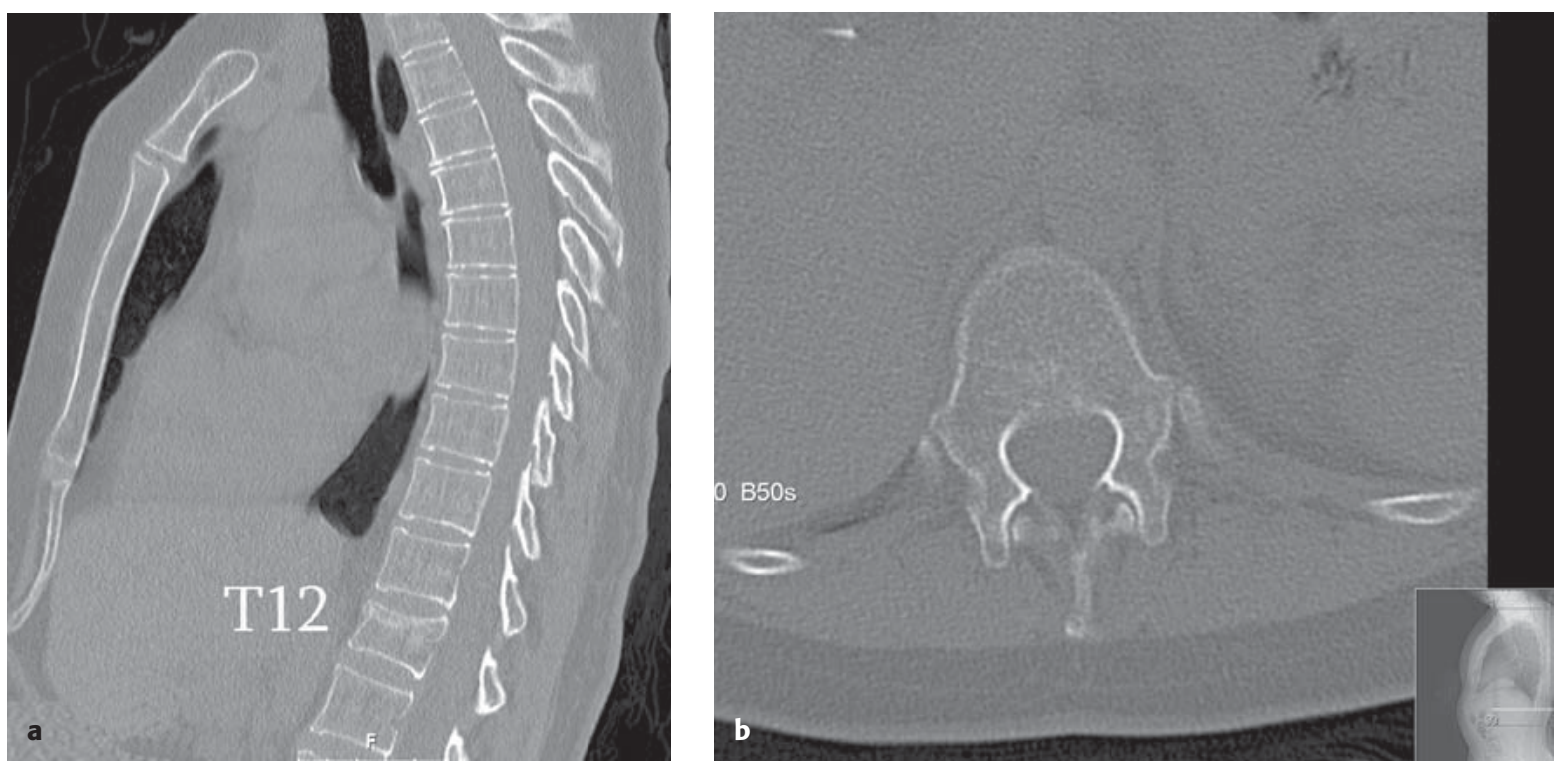
Fracture is a mechanical event that results when the load that is applied to a bone exceeds its ability to bear the load [11]. If the factor of risk is less than 1 , the bone is not predicted to fracture; however, if $\Phi$ is greater than 1, then fracture can be expected [9].

In the clinical cases presented, variables that affect the applied load and fracture load can be analyzed. In the vertebral column, the strongest factors that influence applied load involve the position of the spine and the addition of weight away from the body. Mathematical models developed to predict compressive loads across the thoracolumbar spine demonstrate that loads markedly increase with forward flexion and increasing weight held at a distance from the body's center of mass [9, 12]. The example by Bouxsein et al [13] shows that the force at L2 is 0.5 times the body weight while standing and increases to $1.5 \times$ body weight when the trunk is flexed forward to only $30^{\circ}$ with arms outstretched. With the addition of weight, force magnitudes increase dramatically. Force magnitudes seen across a spine associated with lifting weights of $15-30 \mathrm{~kg}$ are in the $1000 \mathrm{~N}-2000 \mathrm{~N}$ range for a person of average height and weight [9]. In light of the suggested $2154 \mathrm{~N}$ failure load of the elderly vertebrae, one can recognize the increased risk of spine fracture in the aging spine [8]. During a high-speed turn while bobsledding, an additional centripetal force is applied to the bodies of the passengers. The centripetal load is related to the mass and speed of the bobsled as well as the radius of curvature of the turn. This is defined by the equation:

$$
\text { Force }=\text { Mass } \times \text { Velocit }^{2} / \text { Radius }
$$

In the described setting of recreational bobsledding, the applied centripetal force may greatly overshadow body weight forces by orders of magnitude.

Load to fracture is determined by the geometry of the bone, the material properties of the bone, and the loading modality [9]. The geometric changes associated with aging vertebrae include an increase in width at both the endosteal and periosteal surfaces. This expansion results in an increased total area and provides partial protection against fracture [9, 14]. This has been more clearly demonstrated in men than in women and may provide an explanation as to greater severity of injury in the female patient (T12 burst fracture) than the male patient (T12 compression) despite an identical mechanism of injury [9]. Another factor is the material property of the bone. This consists of both cortical and trabecular bone. The most significant change in cortical bone with age is an increase in porosity $[9,15]$. In addition, the toughness of cortical bone declines by $10 \%$ per decade [9]. The age-related trabecular bony changes include, most notably, a decrease in density [9]. The compression strength of vertebral trabecular bone decreases by approximately $70 \%$ from 25 to 75 years of age $[16,17]$. The combination of weakened cortical and trabecular bone contribute to age-related changes in vertebral mechanical properties [16]. The final participating variable is the mode of loading. The loading mode is defined by the direction, type, and speed of load applied [9]. The loading mode in this case is likely a combination of axial compression, forward flexion, and torsional loading. When considering these particular factors, spontaneous nonimpact-derived spinal fracture on a high-speed bobsled run is not surprising. Documentation provided by the engineering firm involved with design and analysis of the track reveals "g forces" in excess of 4 occurring five times during the ride with a peak horizontal "g force" of 4.67. Although called a "g force," this is in reality a measurement of acceleration defined as a multiple of the acceleration of gravity $\left(9.8 \mathrm{~m} / \mathrm{sec}^{2}\right)$. In a simplified model, the forces experience by the rider (assumed to be an average of $70 \mathrm{~kg}$ ) would be up to $3204 \mathrm{~N}$.

$$
\begin{aligned}
& \text { Force }=\text { Mass }(\mathrm{kg}) \times \text { Acceleration }\left(\mathrm{m} / \mathrm{sec}^{2}\right) \\
& \text { Force }=70 \times 4.67 \times 9.8 \mathrm{~m} / \mathrm{sec}^{2}=3204 \mathrm{~N}
\end{aligned}
$$

The subjects in the bobsled were also flexed forward and had an additional weight of a protective helmet. The actual forces experienced by the rider's spine may, therefore, be greater than this estimated value. The patients, in their sixth decade of life, may additionally have age-related bone changes, increasing cortical bone porosity, and decreasing trabecular bone density that may contribute to a weakened load to failure. Combined with large centripetal forces experienced through the bobsled turns, riders are clearly at risk for fracture.

\section{CONCLUSION}

These cases highlight a need for possible safety guidelines or recommendations for recreational bobsledding. Consumers, the managing team at a bobsled track, and the ski patrol or EMT services should be aware of the increasing risk of spine fractures associated with age. It may also be necessary to caution individuals with a diagnosis of metabolic bone disease, renal disease, known ankylosing spinal disorder, and osteoporosis or osteopenia from participating in recreational bobsledding. Additionally, slower velocities during bobsled turns may reduce the risk of spinal fracture and could be considered as an option. While this may diminish some of the subjective "thrill" of the bobsled run, it may make for a safer experience. Beyond the specifics of bobsledding, these cases highlight fracture risks for older individuals participating in recreational sports that might otherwise be thought of as benign. 


\section{REFERENCES}

1. Boden BP, Jarvis CG (2008) Spinal injuries in sports. Neurol Clin; 26(1):63-78.

2. The National Spinal Cord Injury Statistical Center (2009) Spinal Cord Injury: facts and figures. University of Alabama. Available at: www.nscisc.uab.edu

3. Katoh S, Shinghu H, Ikata T, et al (1996) Sports-related spinal cord injury in Japan (from the nationwide spinal cord injury registry between 1990 and 1992). Spinal Cord; 34(7):416-421.

4. Boden BP, Prior C (2005) Catastrophic spine injuries in sports. Curr Sports Med Rep; 4(1):45-49.

5. Ekin JA, Sinaki M (1993) Vertebral compression fractures sustained during golfing: report of three cases. Mayo Clin Proc; 68(6):566-570.

6. Vaccaro AR, Baron EM, Sanfilippo J, et al (2006) Reliability of a novel classification system for thoracolumbar injuries: the Thoracolumbar Injury Severity Score. Spine; 31(suppl 11):S62-S69. Discussion S104.

7. Patel AA, Vaccaro AR, Albert TJ, et al (2007) The adoption of a new classification system: time-dependent variation in interobserver reliability of the thoracolumbar injury severity score classification system. Spine; 32(3): E105-E110.

8. Imai K, Ohnishi I, Yamamoto S, et al (2008) In vivo assessment of lumbar vertebral strength in elderly women using computed tomography-based nonlinear finite element model. Spine; 33(1):27-32.

9. Silva MJ (2007) Biomechanics of osteoporotic fractures. Injury; 38(suppl 3):69S-76S.
10. Myers ER, Wilson SE (1997) Biomechanics of osteoporosis and vertebral fracture. Spine; 22(suppl 24):25S-31S.

11. Hayes WC, Myers ER (1995) Biomechanics of Fractures: Osteoporosis, Etiology, Diagnosis, Management. Philadelphia, Pa: LippincottRaven Publishers: 93-114.

12. Schultz A, Andersson G, Ortengren R, et al (1982) Loads on the lumbar spine: validation of a biomechanical analysis by measurements of intradiscal pressures and myoelectric signals. J Bone Joint Surg Am; 64(5):713-720.

13. Bouxsein ML, Myers ER, Hayes WC (1996) Biomechanics of Age-Related Fractures. Osteoporosis. San Diego, Calif: Academic Press, 373-393.

14. Riggs BL, Melton lii LJ 3rd, Robb RA, et al (2004) Population-based study of age and sex differences in bone volumetric density, size, geometry, and structure at different skeletal sites. J Bone Mineral Res; 19(12):1945-1954.

15. McCalden RW, McGeough JA, Barker MB, et al (1993) Age-related changes in the tensile properties of cortical bone: the relative importance of changes in porosity, mineralization, and microstructure. J Bone Joint Surg Am; 75(8):1193-1205.

16. McCalden RW, McGeough JA, Court-Brown CM (1997) Age-related changes in the compressive strength of cancellous bone: the relative importance of changes in density and trabecular architecture. J Bone Joint Surg Am; 79(3):421-427.

17. Mosekilde L, Mosekilde L, Danielson CC (1987) Biomechanical competence of vertebral trabecular bone in relation to ash density and age in normal individuals. Bone; 8(2): 79-85. 


\section{EDITORIAL PERSPECTIVE}

Aside from the interesting report and cogent commentary provided, the question remains how to manage a 'simple' thoracolumbar compression and burst fracture such as shown in this case.

Both patients were assessed to have intact posterior ligamentous complex structures by review of the CT scans and presumably focused clinical examination. Both were placed in a custom-made thoracolumbar orthosis (TLSO) brace with upright radiographs to check for alignment. They were treated for 6 weeks in such a brace and seemingly did well, despite having sought apparently unauthorized chiropractic care on the side.

There is surprisingly little high-grade evidence for nonoperative care of 'routine' thoracolumbar fractures, both in terms of the type of orthosis chosen and the duration of bracing. A recent review of the literature confirmed the "best practices" standard for nonoperative care to be severely lacking and yet to be defined [1].

This is all the more important as there are increasing comparison trials for vertebroplasty and kyphoplasty using nonoperative treatment comparisons without our profession having a consensus as to how to define appropriate 'nonoperative care.' This nonoperative care approach also remains woefully understudied.

Aside from heightening our awareness of the frailty of the aging human spine under high-impact situations, we need to make further strides in understanding our nonoperative care approach and hopefully standardizing our care in the future.

1. Longo UG, Loppini M, Denaro L, et al (2012) Conservative management of patients with an osteoporotic vertebral fracture: a review of the literature. $J$ Bone Joint Surg $B r$; 94(2):152-157.

\section{COMMENTARY}

Carlo Bellabarba, MD

Dept of Orthopaedics and Sports Medicine

Harborview Medical Center

Seattle, WA, USA

Severson and colleagues describe isolated thoracolumbar fractures in a couple that were caused by an unusual mechanism. Rather than having resulted from a more typical traumatic event, the injuries seem to have occurred due to gravitational forces in which the patients were subjected while descending an Olympic bobsled run. The authors provided an excellent description of the typical forces required to cause fracture in osteopenic vertebrae and a cogent argument as to how this threshold can be exceeded during the bobsled descent that resulted in a near simultaneous "popping" sensation with subsequent back pain in both patients.

The simultaneous aging of our population and increased desire to remain active during later years will undoubtedly result in an increasing number of patients doing activities that subject their vertebrae to forces that exceed their fracture threshold. Although in this particular instance it would be difficult to define the specific risk of fracture caused by gravitational forces sustained during an otherwise uneventful bobsled ride in any given individual, this report is useful in alerting both organizers and participants engaging in such activities of the risk of possible spinal fracture in individuals with known risk factors for compromised bone density, which can in turn allow for a more informed decision as to whether one may choose to participate. 